\title{
An Ethics Framework for Making Resource Allocation Decisions Within Clinical Care: Responding to COVID-19
}

\author{
Angus Dawson • David Isaacs • Melanie Jansen • Christopher Jordens $\mathbb{D}$ • Ian Kerridge • \\ Ulrik Kihlbom • Henry Kilham • Anne Preisz • Linda Sheahan • George Skowronski
}

\begin{abstract}
On March, 24, 2020, 818 cases of COVID-19 had been reported in New South Wales, Australia, and new cases were increasing at an exponential rate. In anticipation of resource constraints arising in clinical settings as a result of the COVID-19 pandemic, a working party of ten ethicists (seven clinicians and three fulltime academics) was convened at the University of Sydney to draft an ethics framework to support resource allocation decisions. The framework guides decisionmakers using a question-and-answer format, in language that avoids philosophical and medical technicality. The working party met five times over the following week and then submitted a draft Framework for consideration by two groups of intensivists and one group of academic ethicists. It was also presented to a panel on a national current affairs programme. The Framework
\end{abstract}

\footnotetext{
A. Dawson - D. Isaacs · C. Jordens $(\bowtie) \cdot I$. Kerridge •

H. Kilham - A. Preisz • L. Sheahan • G. Skowronski

Sydney Health Ethics, The University of Sydney, Medical

Foundation Building (K25), Sydney, NSW 2006, Australia

e-mail: chris.jordens@sydney.edu.au
}

\author{
A. Dawson \\ e-mail: angus.dawson@sydney.edu.au \\ D. Isaacs \\ e-mail: david.isaacs@health.nsw.gov.au \\ I. Kerridge \\ e-mail: ian.kerridge@sydney.edu.au \\ H. Kilham \\ e-mail: henrykilham@gmail.com
}

was then revised on the basis of feedback from these sources and made publicly available online on April 3, ten days after the initial meeting. The framework is published here in full to stimulate ongoing discussion about rapid development of user-friendly clinical ethics resources in ongoing and future pandemics.

Keywords Ethics framework - Resource allocation . Clinical care · Decision-making · COVID-19 .

Pandemic $\cdot$ Australia $\cdot$ New South Wales

\section{Background}

This ethics framework is designed to help clinicians, hospital administrators, and policymakers decide how

\author{
A. Preisz \\ e-mail: Anne.Preisz@health.nsw.gov.au \\ L. Sheahan \\ e-mail: Linda.Sheahan@health.nsw.gov.au \\ G. Skowronski \\ e-mail: mavidica1@gmail.com \\ D. Isaacs $\cdot$ M. Jansen $\cdot$ H. Kilham \\ The Children's Hospital Westmead, Sydney, Australia
}

M. Jansen

e-mail: Melanie.Jansen@health.nsw.gov.au 
to allocate clinical health resources as they become scarce within a pandemic such as COVID-19. Such a framework "frames" decision-making; it does not list or stipulate general answers. Much will be left to expert judgement in response to different circumstances as they arise. Instead of listing a set of abstract values, principles, and rules, this framework aims to help with decision-making by structuring the relevant issues to be considered in the form of a series of questions and answers to ensure it provides relevant and practical guidance.

\section{Part A: Aims of Clinical Practice in Australia}

These aims are high-level and overarching across all clinical activities, including COVID-19 planning and response. The aims can guide us as we work through the questions and answers that follow. In stating these aims, we are assuming that the following are at least implicit and sometimes explicit aims of clinical practice in Australia:

Aim 1. Work with patients to deliver appropriate healthcare in response to their needs.

Aim 2. Ensure the continuity of a sustainable healthcare and public health system.

Aim 3. Promote the maintenance of trust in the health system.

Aim 4. Where appropriate, conduct research and innovation to improve patient care.

Any ethics framework for clinicians should be compatible with, and should further, these aims.

\section{Part B: A Framework for Resource Allocation Decisions in Response to COVID-19}

Many ethical questions arise in clinical care. Ethical issues that routinely arise, for example relating to

I. Kerridge

Royal North Shore Hospital, St Leonards, Sydney, Australia

U. Kihlbom

Centre for Research Ethics \& Bioethics, Uppsala University, Uppsala, Sweden consent and confidentiality will, of course, continue to be relevant. This framework aims to provide practical advice for resource allocation decisions that will arise in the delivery of healthcare as a result of the COVID-19 pandemic.

\section{What Is Resource Allocation?}

When the supply of something is inadequate to meet demand, we must decide how to distribute it. Resource allocation is the mechanism we use to do this. Examples of essential resources that we can expect to be limited during the COVID-19 pandemic will include ventilators, access to Intensive Care Units (ICU), clinical expertise, personal protective equipment (PPE), COVID19 testing, medications, and vaccines.

Resource allocation decisions necessarily involve ethical considerations. Choices that are made, including doing nothing, will impact on, and may even end, people's lives. The main ethical issue in resource allocation is ensuring that any decisions that we make are fair or just. How we decide this will be contentious and difficult. (Aims 2 and 3).

\section{Can All Resource Allocation Decisions Be Made in Advance?}

No. Whilst we can think about and discuss issues in advance, decisions will inevitably have to be responsive to individual situations of scarcity. For example, decisions about allocating PPE will be different from those about allocating vaccines. Decisions about the same resource might differ between hospitals or even within the same hospital over time. We do, however, have an obligation to plan in advance, based upon the best possible understanding of the characteristics of the virus (e.g., means of transmission, who is most at risk, etc.)

\footnotetext{
A. Preisz

The Sydney Children's Hospitals Network, Sydney, Australia

L. Sheahan · G. Skowronski

St George Hospital, Sydney, Australia

L. Sheahan

South East Sydney Local Health District, Sydney, Australia
} 
and what we know about the amount of each resource. Such planning provides the basis for using our resources in the most efficient way possible. (Aims 2 and 3).

\section{What Processes Should We Adopt for Making Resource Allocation Decisions?}

Resource allocation decisions will always be controversial. However, we can agree on a process for reaching decisions, even if we disagree on which ethical considerations we should use. Decisions are more likely to be accepted by individuals, clinical teams, organizations, and the public, where they can see that the decision-making process is a fair one.

The characteristics of a fair process can include such things as being clear and open about decisions, the reasons for those decisions, who made them, and the possibility of revising decisions in the light of new evidence or new relevant considerations. In ideal circumstances groups affected by the decisions should be consulted and opportunities for formal appeals against decisions should be provided. However, this will not always be possible because, for example, decisions will often need to be made quickly in response to rapidly changing circumstances. (Aims 2 and 3).

\section{Who Will Make These Decisions?}

We suggest that a group is put in place in each institution to make pandemic allocation decisions. Each institution will have to consider the kind of membership that will work best for them. The key aim is to separate out care and advocacy for a particular patient from the allocation decisions. This provides a level of detachment from the immediate clinical needs of each patient, better ensuring a clear and defensible process, thereby reducing the opportunities for accusations of bias. It also has the advantage of protecting those clinicians caring directly for patients from some of the direct stress and anxiety if they are the ones to be the decision-makers about allocation. (Aims 2 and $3)$.

\section{What Are the Relevant Justifications for Resource Allocation Decisions?}

Three main ideas are often suggested.

(a) Equal value to all: It is almost universally agreed by clinicians and the public that each person is due equal respect or is equally valuable. This means that we should not use characteristics such as gender, ethnicity, sexuality, disability, religious or political views etc., as the basis for allocation decisions. To do so would be discrimination. Many would also hold that we should not discriminate on the basis of wealth or ability. However, we should note that entirely avoiding all such discrimination is impossible because a person's current health status to some degree reflects socioeconomic determinants of their health.

(b) Getting the most out of resources: We could give priority to interventions that get the most out of our limited resources, where this is interpreted in slightly different ways (e.g., most efficient use of resources, most medical benefit, most costeffective use, giving priority to healthcare workers so they are able to continue working and benefit the population as long as possible, priority to the young over the old to maximize length of lives saved, priority to those with care responsibilities).

(c) Giving priority to those in need: We could give priority to those individuals or groups who are seen to need restoring to some level that is held to be appropriate, a threshold which they currently fall below (e.g., priority to those in greatest medical need, priority to the worst-off or most disadvantaged, priority to those at increased risk of harm, priority to those we know have suffered injustice in the past).

Discussion of the allocation of scarce resources in a pandemic often appeals to one or more such considerations, expressed in terms of a range of values, rights, principles, and theories. (Aims 1, 2, and 3).

\section{How Should We Decide How to Allocate Scarce Resources?}

Drawing on the discussion above, we state that once resources are scarce our primary obligation is to ensure 
that we gain the best value we possibly can from the expenditure of that resource. This view is controversial, but it can be justified on two grounds. First, we can appeal to many of the different justifications included in the answer to question 5 (i.e., getting the most out of resources, as well as acting to restore people to a healthy state, etc.). Second, if an allocation process fulfils this primary obligation, and it meets the procedural conditions described in answer to question 3, then we can argue that this also fulfils our commitment to accord equal value to all. This is because such a process of allocation decision-making begins with the idea that resources are, in fact, scarce. Scarcity of a resource will, sooner or later, create a "tipping point" where decisions are no longer made as they normally are — on the basis of clinical need alone. Decisions after that point should be guided by a different system of allocation because we cannot provide the resource to all.

How, exactly, "best value" applies will differ in each set of circumstances and must be considered on a case-by-case basis. It is for each organization to plan their system for resource allocation. We cannot possibly capture all of the different factors that will be relevant. However, it might be helpful to consider an example that illustrates the kinds of things that may be considered relevant. Our example is the allocation of access to the Intensive Care Unit (ICU) - including access to ventilatorsduring the COVID-19 outbreak. This is one kind of triage system: a formal system of allocation that operates once we can no longer act to save everyone.

\section{Step 1: Defining the "Tipping Point"}

At some point in the epidemic, a consensus will emerge that scarcity exists for a particular resource in a particular location. This creates a "tipping point" where decisions are no longer made under the conditions of normal care because there is a requirement to implement "best value" use of the relevant resource due to that scarcity. Clinicians should be careful not to "tip" into this mode too early, when scarcity does not yet exist, as this potentially compromises patient care. Scarcity may exist for one resource (ventilators) but not another (PPE) and it may be temporary, as we await expected re-stocking, or for the foreseeable future. This should be reviewed constantly as resource levels fluctuate.
Step 2: Is the Patient Eligible for the ICU or not?

This is the start of the triage process for all patients who are candidates for possible ICU admission (whether they have the SARS-CoV-2 (COVID) virus or not). Three groups of patients are to be excluded from consideration for ICU:

- The first group is those who are likely to recover. They are expected to survive without access to the ICU, even if they would, in normal circumstances, be admitted and could benefit from it.

- The second group are those who are dying. According to the best available evidence they are terminally ill (e.g., they have advanced, inoperable cancer). They are to be given supportive care, including palliative care, but are not to be considered for ventilation, etc.

- The third group are those who choose not to be admitted to the ICU.

Those patients that are judged to be eligible for access to the ICU during a pandemic must meet the medical criteria used for admission to the ICU in normal times and they should not be in one of the exclusion groups. They go on to the next step.

\section{Step 3: How Should We Prioritize Patients who are Eligible for Admission to ICU?}

How exactly this is done may depend on local circumstances. To simplify, we will consider allocation to two main groups:

(a) High Priority: Those who, on the basis of their current medical condition, are highly likely to recover and would benefit long-term from admission to the ICU.

(b) Low-Priority: Those who, on the basis of their current medical condition, may recover after admission to the ICU.

This is, mainly, a medical judgement, and as such it will take into account multiple factors about each individual patient, including the medical condition of the patient, their expected tolerance of the treatment, the expected outcomes of treatment, the existence of comorbidities where these are relevant to expected benefit, etc. Decisions should not be made simply on the basis of 
age, but other things being equal, length and quality of expected post-ICU life will be relevant. These overall judgements are to be driven, ultimately, by the key idea of "best value" use of any resource. Some will find this unacceptable, because some patients with pre-existing morbidities will be disadvantaged (where, in many cases, their health status is shaped by previous socioeconomic determinants). However, we believe we are obliged to focus on using scarce resources as efficiently as possible, and at this crucial point we must focus on "best value." For those that disagree, the most plausible alternative allocation method is using some kind of lottery system. Such a system may respect each person by giving them an equal chance of access to the resource in question, but overall it will result in less efficient outcomes.

\section{Step 4: How do We Prioritize Within the "High Priority" Group?}

If resources are very scarce, then we may need to prioritize within the "high priority" group. Here, other factors, beside those listed in Step 3, may be used to rank patients. These can be considered "tie-breaker" considerations, that is, they come into play when patients are otherwise equally eligible for admission to the ICU on medical grounds, but there are not enough ICU beds to accommodate them all. Any such ranking needs to be openly declared and defended within the particular system set up by the relevant organization.

Some of these further considerations may be justified through appeal to the "best value" criterion, where it is interpreted as extending beyond the individual patient. For example, clinicians who are ill with COVID-19 might receive priority as they could re-join the fight against COVID-19. They might be particularly useful if they have immunity. However, if they have received ventilator support, we might doubt that they will actually be able to offer much assistance in the course of this outbreak (unless it extends for many months). Other possible groups that we could choose to prioritize might include pregnant women or those with caring responsibilities for others (e.g., children or elderly relatives, etc.).

We might also choose to prioritize patients from First Nations communities. Here the justification could be due to existing disadvantage, what we know of poorer outcomes for these groups from previous outbreaks, and some compensation for previous sustained injustice.
Because these considerations are not based purely on medical grounds relating to the individual patient, they are likely to be contentious (as stated above under Question 5). This is why they need to be clearly articulated and clearly justified.

It is important to note, again, that some grounds for prioritizing are inappropriate, such as those that may be discriminatory (see the answer to Question 5).

\section{Step 5: Regular Review}

Patients who are admitted to ICU are regularly reviewed by clinicians. There is a temptation to spend a lot of time developing metrics that allocate sophisticated scores to be given on the basis of patient characteristics. We suggest that it is more important to have a flexible process that allows for daily evaluation of each patient, followed by reallocation of resources as necessary. For example, where a patient who was previously expected to recover without ventilator support declines and a ventilator is indicated, they are to be reassessed along with everyone else. On review, patients who are not responding to treatment may have their treatment downgraded (reverse triage). It will be possible that, given the scarcity of resources, some patients will be removed from ventilators earlier than they would be in usual circumstances. Again, any relapse will require evaluation within the context of all patients that are eligible for ventilation. This follows the "best value" criterion for resource use and rules out considerations such as incumbency and "first come, first served" as irrelevant. (Aims 1, 2 and 3).

\section{Should We Prioritize Patients with COVID-19 Over Patients Without COVID-19 in Resource Allocation?}

No. The key idea, at times of resource constraint, is to focus on the "best value" use of the relevant resource (as defined in the answer to Question 6 above). There are no good grounds to prioritize patients with COVID-19 over those without COVID-19. However, given the emerging pandemic we should prioritize responding to the pandemic over all other non-essential interventions that can be postponed such as elective surgeries. Where 
patients without COVID-19 are discharged earlier due to the pandemic, they should receive more extensive continuing care at home to ensure they are not disadvantaged (Aims 2 and 3).

\section{Should the Standard of Care for Patients Change in an Epidemic?}

The primary obligation of every clinician - to do what is best for their patients-will remain in place during a pandemic. However, the range of options for what is "best" will change as a result of the constraints imposed by the pandemic. For example, patients may not be allowed to have visitors (especially when PPE is scarce). This is necessary to protect staff and other patients, and reduce the risk of transmission from and to visitors. This could mean that some patients become seriously ill and die without the usual support of relatives and friends. This will inevitably cause distress to all parties. Access to other modes of communication such as phone and video calls should be provided where possible. As set out in reply to Question 9 below, it is important for healthcare organizations to inform the public about changes to standards of care. (Aims 1, 2, and 3).

\section{What Are the Obligations of Organizations?}

It is essential that organizations such as hospitals provide safe systems of work as a means to protect the physical and mental health of their staff. For example, there is an obligation to enforce safe rostering, even if individuals wish to work more hours. This is especially important in a pandemic that is likely to last many months and is not just a one-off emergency event (such as a major car crash). Organizations are obliged to ensure that the hospital is kept free of infection through cleaning, as part of the provision of a safe environment for all patients, volunteers, and staff, including cleaners, porters, and ancillary staff. Organizations should also think of developing policies that provide for greater staffing resources, a key element in responding to COVID-19. For example, where clinicians from other areas are redeployed, or student and retired clinicians are called into work, extra training may be needed, or limitations may have to be placed upon availability for some tasks or restrictions on hours to be worked.

Organizations have a key role to play in providing information about resource allocation to the public. Many of the answers to questions above emphasize the importance of procedures such as open communication of the system for decision-making and how individual decisions are reached. Once systems for resource allocation are in place, these can be outlined to the public. Truthful communication may be difficult and at times uncomfortable, especially with controversial issues. Organizations have an obligation to ensure that they have staff with relevant media training to assist with such messaging and ensuring coordination with public health and state authorities. (Aims 1, 2, and 3).

\section{What Are the Obligations of Individual Clinicians?}

Clinicians involved in responding to COVID-19 should accept that they are, themselves, a vitally important and scarce resource that also needs to be used wisely. Clinicians should protect themselves and not work in circumstances where they are at unnecessary risk (e.g., where there is inadequate PPE). Clinicians should avoid "heroic" or "sacrificial" actions that are likely to take them out of the healthcare system (e.g., entering a room without PPE to resuscitate a patient who is known to be infected). This is especially important when clinicians have particular specialist knowledge, such as experience maintaining ventilators. Actions should be informed by the best evidence and a general obligation to use all resources wisely. For example, patients should not be tested when it is contrary to recommendations (given a shortage of tests), nor should they waste PPE on low-risk procedures and patients. Clinicians have obligations not just to each individual patient, but also to themselves, their family, other patients, other clinicians, and to society more broadly. (Aim 3).

\section{Can Research Be Conducted During an Outbreak?}

Yes. It is vitally important to better understand the nature of a particular outbreak, as well as potential 
interventions and changes that might be beneficial to patients and families. However, research should never impede clinical care. In fact, many of the resources that would otherwise be used in research will inevitably be diverted into clinical care during a pandemic. Some argue that those who participate in COVID-19 research should then receive greater priority in access to relevant interventions. If this is accepted, then research participants may have to be added to the list contained in the answer to Question 6. (Aims 2 and 4).

Publisher's note Springer Nature remains neutral with regard to jurisdictional claims in published maps and institutional affiliations. 